\title{
Viewpoints: how the hippocampus contributes to memory, navigation and cognition
}

\author{
John Lisman, \\ Department of Biology at Brandeis University, Waltham, Massachusetts, USA \\ György Buzsáki, \\ NYU Neuroscience Institute at New York University, New York, New York, USA \\ Howard Eichenbaum, \\ Center for Memory and Brain at Boston University, Boston, Massachusetts, USA \\ Lynn Nadel, \\ Department of Psychology and Cognitive Science Program at University of Arizona, Tucson, \\ Arizona, USA \\ Charan Ranganath, and \\ Center for Neuroscience and Department of Psychology at the University of California, Davis, \\ California, USA
}

\section{A. David Redish \\ Department of Neuroscience at the University of Minnesota, Minneapolis, Minnesota, USA}

\begin{abstract}
The hippocampus serves a critical function in memory, navigation, and cognition. Nature Neuroscience asked John Lisman to lead a group of researchers in a dialog on shared and distinct viewpoints on the hippocampus.

There has been a long history of studying the hippocampus, but recent work has made it possible to study the cellular and network basis of defined operations-operations that include cognitive processes that have been otherwise difficult to study (see Box 1 for useful terminology). These operations deal with the context-dependent representation of complex memories, the role of mental exploration based on imagined rather than real movements, and the use of recalled information for navigation and decision-making. The progress that has been made in understanding the hippocampus has motivated the study of other brain regions that provide hippocampal input or receive hippocampal output; the hippocampus is thus serving as a nucleating point for the larger goal of understanding the neural codes that allow inter-regional communication and more generally, understanding how memory-guided behavior is achieved by large scale integration of brain regions. In generating a discussion among experts in the study of the cognitive processes of the hippocampus, the editors and I have posed questions that probe important principles of hippocampal function. We hope that the resulting discussion will make clear to
\end{abstract}

Correspondence should be addressed to J.L. (Lisman@brandeis.edu) or A.D.R. (redish@umn.edu).

Competing Financial Interests: The authors declare no competing financial interests. 
readers the progress that has been made, while also identifying issues where consensus has not yet been achieved and that should be pursued in future research. - John Lisman

\section{What is the global function of the hippocampus and how does this relate to the concept of a cognitive map?}

We know that the hippocampus is required for episodic memory, but the role of spatial information, so obvious in rodent recordings (for example, place cells), remains to be clearly defined. Regions that provide input to the hippocampus (Fig. 1) appear to be capable of encoding a great deal of information about space (for example, parietal and parahippocampal cortex), even in the absence of a functioning hippocampus. What, if any, is the unique contribution of the hippocampus and how does this relate to the concepts of episodic memory and cognitive map?

\section{Nadel and Ranganath}

There is near consensus that the hippocampus is essential for the spatial representation of environments and for the ability to remember specific events, or 'episodic memory' ${ }^{1}$. The hippocampus supports these abilities by providing a spatial and temporal framework for relating experiences, creating a 'cognitive map' of the organism's experienced world. Because research in rodent models has focused on the characteristics of place cells, the emphasis on space became the default way of thinking about the hippocampal cognitive map. However, as Eichenbaum points out, O'Keefe and $\mathrm{Nadel}^{2}$ originally conceptualized the hippocampal cognitive map as providing a spatial and temporal context in a manner that supports memory for both spatial layouts and past episodes.

The distinctive contribution of the hippocampus to space relates to the fact that it represents spatiotemporally coincident elements, the pieces and patches of experience, in an 'allocentric' ${ }^{2}$ or 'relational' ${ }^{3}$ framework that is independent of the current location of the observer in time and space (for instance, the hippocampal representation might be "the clock tower is north of the bridge" or "He crossed the bridge before he went to the clock tower" see, for example, Deuker et al. ${ }^{4}$ and Nielson et al. ${ }^{5}$ ). By separating the elements of past events from one's current time and place (e.g., by replaying in place A an event that occurred in place B), the hippocampus provides a form of representation that can be used in retrospective (i.e., episodic memory retrieval) or prospective (i.e., prediction or simulation) cognition. Although spatial information is present in hippocampal input regions, the utilization of this information to create navigable and annotatable maps appears to first occur in the hippocampus.

Hippocampal maps are inextricably linked to spatial and episodic contexts (Fig. 2). A spatial context is typically operationalized as an area that is delineated by environmental boundaries, and hippocampal place cells map relative locations within the spatial context ${ }^{6,7}$. Importantly, a given physical area can be represented (at different times) by different maps if the behavioral or sensory situation is sufficiently different. An episodic context, in turn, can be operationalized in terms of chunks of time that are defined by a behavioral goal or situation $^{8}$, and what have been referred to as hippocampal time cells map time within an 
episodic context ${ }^{9,10}$. It's important to point out that place cells are sensitive to episodic context: for instance, cells that encode particular locations as an animal travels from point A to point $\mathrm{B}$ can encode entirely different locations when the animal runs from point $\mathrm{B}$ to point $\mathrm{A}^{11-13}$. Thus, the hippocampus constructs multiple maps with overlapping content. Because only one map can be active at a given moment, sensory information and information about goal states are used to either activate a pre-existing map or to create an entirely new map.

Context-dependent coding is a critical property that explains how the hippocampus contributes to behavior. In a novel context, the organism may engage in exploration before taking further action. In a familiar context, the organism can bring its past experience in the context to bear on upcoming decisions and actions. Violations of predictions formed from past experiences in the context could lead to the formation of an entirely new context representation, or alternatively, the new information could be accommodated within an already existing context map-these phenomena are currently being studied under the rubric of 'memory reconsolidation' ${ }^{14}$, 'memory updating ${ }^{15-18}$, and 'integrative encoding' ${ }^{19}$. In other words, hippocampal context representations can stimulate exploratory behavior, inform decisions from past experience, or rapidly update our understanding of the current state of the world.

\section{buzsáki}

Let me try to approach potential answers from two different directions. First, does the hippocampus perform a global or generic computation? Second, does the hippocampus solve a cognitive problem that the investigator can view as global or general?

From the computational point of view, one may consider the hippocampus as a generalpurpose sequence generator that encodes content-limited ordinal structure and tiles the gaps between events or places to be linked ${ }^{20,21}$. This may be viewed as a global function. In support of this general mechanism, patients with hippocampal damage can recall considerable spatial and temporal details of previous journeys but not their sequential $\operatorname{order}^{22}$.

Approaching the question from outside-in, one should know that the hippocampusentorhinal system has a topographically organized bidirectional communication with the large neocortex (Fig. 1). During the course of mammalian evolution and the corresponding disproportional enlargement of the neocortex, hippocampal inputs shifted from largely sensory, spatial, and motor representations in the rodent to interactions mainly with higherorder cortical areas in primates. The hippocampus itself can be considered as a single giant module, composed of several layers (dentate gyrus, CA3, CA2, CA1) in which the large recurrent axon collateral system of the CA2-CA3 regions mix and segregate the input messages without any special consideration of their source. It is, therefore, expected that, whatever information is presented to the hippocampus from whichever parts of the neocortex, the same general computational algorithms will be performed. In other words, the hippocampus may be 'blind' regarding the modality and nature of the inputs. It processes the sent messages the same way irrespective of their origin and returns its judgment to the source. Thus, given the many possible neocortical routes to the hippocampus, the answers to the question, "what is the function of the hippocampus?" may be different depending on the 
routes the investigator tests in her experiment. It may appear to be space, time, sound frequency, odor-sound sequence, memory, or something else, even though the hippocampus will respond to each case by generating unique cell-assembly sequences relevant to the particular situation.

\section{Eichenbaum}

In The Hippocampus as a Cognitive Map, O'Keefe and $\mathrm{Nadel}^{2}$ introduced their thesis by stating (p. 1), "We shall argue that the hippocampus is the core of a neural memory system providing an objective spatial framework within which the items and events of an organism's experience are located and interrelated." I endorse this characterization of the network mechanisms of the hippocampus fully, but at the same time, I realize that the role of the hippocampus must be extended beyond a framework of physical space, because a restriction to space fails to account for many observations from studies of the firing properties of neurons in animals, functional imaging in humans, and the effects of hippocampal damage in both animals and humans. In particular, there is a vast literature showing that humans depend on the hippocampus for specific types of nonspatial as well as spatial memory (called declarative and relational) and that the hippocampus activates when humans encode or retrieve these same types of spatial as well as nonspatial memories. To bridge the gaps between behavioral analyses in animals and humans, over the last 30 years I have developed novel behavioral models to show that rats also depend on the hippocampus for memories of relations (including temporal relations) among memories and for flexible use of nonspatial memories in situations beyond repetition of the learning experience. Furthermore, I and others have shown that hippocampal neurons encode events in spatial and nonspatial (including temporal) contexts and in relation to each other, thus merging the features of relational memory with Tulving's conception of episodic memory ${ }^{1}$ as the ability to recall events in spatial and temporal context. Now, a large body of emerging evidence demands that a broader view of hippocampal cognitive maps must be adopted. Specifically, hippocampal relational representations are maps of cognition, not maps of physical space, providing neural network mechanisms for Tolman's broad conception of cognitive maps as well as Tulving's definition of episodic memory.

\section{Redish}

There seem to be four computational functions that hippocampus is playing: (i) enabling bridging across shifts in context, allowing one to recognize when one has returned to a familiar context and where one is within that context, (ii) providing a map associating internal (self-motion, dead reckoning) and external (sensory) cues, which can be used for (iii) search processes on that map for planning, deliberation, and the identification of novel paths and connections, and finally (iv) providing a resource for consolidation. All of these processes have extensive literatures supporting them, and both spatial and contextual information would be useful for all of them. Moreover, computational models suggest that these processes can coexist within the same neural circuitry. Rather than suggesting that hippocampus has a single global function, I think we should think of it as performing multiple computations on a specific representation. That representation is well-described as an encoding of the relationships between external sensory objects and an internal coordinate 
system, in other words, a cognitive map $2,23,24$. Importantly, those relationships include information about sequences, providing both spatial and temporal context.

It is also important to look not just at the hippocampus but also at other structures and to note what is being represented within them. For example, although striatum can show spatial representations on some spatial tasks, those representations only occur when space is informative of reward ${ }^{25,26}$, suggesting that the striatum is actually encoding situation-action pairs, more consistent with its role in procedural memory and decision-making processes. Similarly, although space can be decoded from medial prefrontal cortex (for example, prelimbic cortex) firing, the representational patterns of the prefrontal cells are better described as encoding subtask components within a behavior ${ }^{27,28}$.

\section{Is there clear experimental evidence that the spatial information observed in the hippocampus is actually used in navigational decisions?}

\section{Nadel and Ranganath}

Rodent physiology studies and human imaging studies provide convincing evidence that the hippocampus represents key spatial information during both virtual and real navigation $^{2,8,29-31}$. Absent the hippocampus, nonhuman animals make poor navigational decisions, at minimum, or just seem lost altogether. Moreover, there is a strong relationship between primate hippocampal function and exploration and navigation of a spatial context through eye movements ${ }^{32,33}$. Hippocampal activity is tightly related to the influences of memory on oculomotor exploration ${ }^{33-36}$, and damage to the hippocampus reduces spontaneous oculomotor exploration and eye-movement-based indices of spatial and contextual memory ${ }^{37,38}$. Damage to the hippocampus also disrupts the perception of subtle spatial relationships amongst elements of scenes 39,40 .

Having said that, it is clear that extrahippocampal regions, such as parahippocampal, retrosplenial, and medial prefrontal cortex (and possibly even posterior cingulate and the precuneus), also contribute to spatial memory and navigation ${ }^{41-43}$. These regions, along with key subcortical nuclei in the anterior thalamus and mammillary bodies, form a tightly interconnected anatomical network ${ }^{44}$. Thus, it is not surprising that humans with hippocampal damage can show considerable preservation of spatial behavior, though their performance is not completely normal ${ }^{45,46}$. For instance, the amnestic taxi driver reported by Maguire et al. ${ }^{47}$ retained a considerable degree of knowledge about relative locations of landmarks in London, but he failed at navigation when he had to rely on a detailed representation of the environment.

Overall, it would be inappropriate to say that the hippocampus is necessary for navigation in all situations, because navigational decisions can be driven by multiple, redundant representations (as Redish also notes). Nonetheless, the hippocampus contributes to the precision of navigation, by providing a systematic frame-work to relate landmarks with one another. 
In order to answer this question, we first have to define both decision and navigation. If we define decision as action selection and navigation as action selection through space, then the answer to whether hippocampal spatial information is used in navigation decisions is "sometimes". This goes back to the points made by O'Keefe and Nadel': there are some actions taken through space that are hippocampally dependent and some that are not. In modern theories, there are at least three neurally separable action-selection systems: an instinctual system (often called 'Pavlovian') in which one learns when to release a prewired action, dependent on amygdala, a habit system (often called 'procedural') in which one learns arbitrary situation-action chains, dependent on dorsolateral striatum, and a planning system (often called deliberative') in which one searches through future outcomes to find the best action (see ref. 48 for review). This deliberative planning system can find new paths on a cognitive map. Current theories suggest that the deliberative planning system depends on interactions between hippocampus and prefrontal cortex.

It is clear that hippocampal representations are critical when recognizing spatial contexts after a change (such as in the water maze or contextual fear conditioning) and that hippocampal representations are critical when coordinates have to be realigned (such as in response to shifting goals; see ref. 24 for review). Furthermore, in tasks in which decisions depend on knowing one's position relative to a shifting goal, rodents are only able to find the goal or avoid the shock when the spatial representation in hippocampus is aligned to that goal's coordinate system ${ }^{49,50}$. An interesting question is whether hippocampus is necessary for deliberative, planning actions. Extensive evidence certainly shows that hippocampal disruption shifts decision systems away from deliberative planning systems to the other two systems (see ref. 24 for review). Moreover, disruption of hippocampus leads to changes in these systems' ability to make those decisions. For example, on a task in which rats alternated between a working-memory-dependent journey (go to where you didn't just come from) and a non-working memory-dependent journey (only one option to go back to), Jadhav et al. ${ }^{51}$ found that transient disruptions of hippocampus impaired rats' ability on the working-memory-dependent journey but not the non-working memory-dependent journey, implying an inability to use that memory in decision-making.

At this point, however, no one has directly connected the hippocampal representations during behavior to goal locations with navigation decisions using causal methods. I suspect that making this connection will require manipulations of nonhippocampal structures in response to hippocampal information. For instance, starting from the well-established data showing that hippocampal cells reactivate during sleep and that this is critical for memorybased behavior the next day, de Lavilleon et al. ${ }^{52}$ stimulated dopamine signals when a given hippocampal cell (with a place field that had been measured previously) was active during sleep and found that the location of that cell's place field had become a goal for the rat the next day, implying that the spatial information in hippocampus is used in consolidating navigational information during sleep. 


\section{Eichenbaum}

It is essential to understand what navigation is and to understand the distinction between a cognitive map and navigation. Despite their obvious importance to the study of the neurobiology of navigation, these distinctions are rarely considered in place and grid cell research, especially when only one type of navigation is attributed to using a cognitive map. Navigation is the process of getting from start to finish ${ }^{53}$, and there is broad consensus among cognitive psychologists, animal behaviorists, and roboticists that there are several ways to navigate ${ }^{54}$. Open-field foraging and running on linear tracks, as commonly used to study the spatial firing properties of hippocampal and entorhinal neurons, do not involve strong navigational demands because animals walk in random paths or simply approach visible targets in these tasks. Thus most of the data on place and grid cells are irrelevant to navigation, although these properties could provide a substrate for a spatial map and do predict spatial choices. Indeed, another prominent navigational strategy is to read out a map, either one on paper or your GPS or the spatial cognitive map in your head, and use the remembered map plus memories of experiences in the environment to guide you from start to finish (called 'survey navigation'). I believe the observations of place cell sequences linked to spatial decision-making ${ }^{55}$, and the firing patterns associated with directions, boundaries, routes, and other spatial aspects of memories contribute strongly as elements of memories of spatial behavior in the environment. In addition, the observation of differential activity of place cell sequences as rats traverse the over-lapping segments of different routes through a T-maze (called 'splitter cells') ${ }^{56,57}$ indicates that the rat hippocampal network also supports navigation by following distinct paths even as they pass through some of the same locations (called 'route following'). In real-world navigation (for example, sailing) people bring a vast array of skills and knowledge to get from here to there ${ }^{58}$, and the hippocampus most strongly activates in association with events of memory processing, not just passing through places in a map, during virtual navigation in human ${ }^{59}$. Correspondingly, the essential role of the hippocampus in rodent navigation disappears when the demand for memory is removed, in the water maze when the goal is flagged (see work from Richard Morris) and in T-maze alternation when the delay between trials is eliminated ${ }^{56}$.

\section{Buzsáki}

In an experiment specifically addressing this question, the color of the experimental apparatus was changed across trials. As a result, hippocampal neurons 'remapped' (Fig. 2), that is, different assemblies were active in different contexts. Yet despite the different hippocampal maps, navigational decisions were not impacted ${ }^{60}$. Conversely, 'scrambling' the temporal relationships (at theta-gamma time scale) among spikes of hippocampal place cells by activating cannabinoid receptors did not affect place field relationships. Despite the intact place map, the rat's navigational performance fell to chance ${ }^{61}$.

These phenomena should be understandable by direct observation of known hippocampal output signals, but that understanding has not yet been achieved for several reasons. Most neuroscience research assumes that information is 'coded' if a correlation between external stimuli and neuronal activity in a particular brain region is identified by the experimenter. This is an insufficient, experimenter-designed test. Instead, we must ask how neurons in the down-stream target regions read out hippocampal spiking activity to eventually drive overt 
behavior. Without such 'grounding', the human experimenter remains the interpreter and arbitrator of all correlations between real-world events and neuronal activity. Recreating physiological assembly sequences in the same context in order to elicit the same overt behavior would be useful step in this direction ${ }^{52}$. Remarkable progress has been made toward this goal using optogenetic activation of those very neurons that were activated by task variables, albeit in simple situations where the choice repertoire is limited (for example, to freeze or not to freeze $)^{62,63}$. A more rigorous test would be to understand the transformation rules (how input patterns generate outputs patterns) between the hippocampus and target structures (for example, subiculum and lateral septum), recreate the response patterns in these target areas, and demonstrate that such a manipulation is followed by the appropriate behavioral response.

\section{Do hippocampal pyramidal cells reliably encode nonspatial information?}

Does the hippocampus play a restricted role in encoding spatial and/or temporal contexts, or does its reach extend to literally any kind of information (for example, objects, internal states)? According to the concept of rate-remapping, nonspatial sensory information is encoded by the rate modulation of place cells. If this concept is valid, doesn't it support the idea that 'place' has a special role in the hippocampal coding?

\section{Eichenbaum}

Tulving's distinction between episodic and semantic memory has been applied to the brain with the proposal that the hippocampus is specifically involved in episodic memory, defined as the ability to remember specific events in spatial and temporal context ${ }^{64}$. Vargha-Kadem et al. ${ }^{65}$ provided evidence cited in support of a special role of the hippocampus in episodic memory by showing that humans with transient anoxia around birth develop lasting impairment in episodic memory while nonetheless being able to acquire general world knowledge over the course of their schooling.

Evidence from recordings of hippocampal neurons is entirely consistent with the notion that the hippocampus is involved in the episodic encoding of events, including the representation of both space and time, and more. When rats must remember an odor during a recent experience, hippocampal cells equivalently code information about encountered odors and about their spatial contexts ${ }^{13}$. Other evidence, discussed in more detail in the next question below, shows strong coding of place and time, separately, as revealed when the other of these two dimensions is controlled. When animals are moving repeatedly through specific paths defined by sequences of places across time (for example, directional running on a track), place cell activation is linked to the temporal order of places traversed (directional place cell activity). Other behavioral variables are also incorporated into hippocampal coding of space and time when they are task-relevant, the most prominent of which is the direction of movement; but velocity is also encoded (see work from Bruce McNaughton), as are, as noted above, specific stimuli that are encountered during the task and must be remembered ${ }^{13}$.

A variety of cognitive manipulations, including training on specific trajectories ${ }^{56,57}$ (and see work by Etan Markus), reward and fear associations (see work by Sheri Mizumori and Isabel 
Muzzio), and motivational states (see work from Pamela Kennedy and Matthew Shapiro), result in a combination of rate and global remapping of place cells and time cells. The claim that manipulation of nonspatial cues is linked exclusively to rate remapping is not supported consistently across studies. An example comes from studies involving training events that all involve identical environmental cues. In some of these studies, spatial coding is globally affected, as measured in terms of low spatial correlations of cell firing fields between conditions, by training on nonspatial cues in the same environment, for example, fear conditioning in the same environment (see work by Sheri Mizumori and Isabel Muzzio) or different navigational strategies in the same maze (see work by Etan Markus). Note that with regard to the Leutgeb et al. study ${ }^{66}$, which identified rate mapping as the selective response to manipulation of nonspatial (color, shape) cues, previous high-profile studies have shown that exclusive manipulation of those same cue dimensions caused global remapping or a mix of rate and global remapping 7,67 .

The observation of global remapping is sometimes interpreted as merely reflecting a change in the animal's frame of reference, but the inclusion of reference frame in the hippocampal coding of space goes beyond any purely place-based representations. Other variables would seem to be in play in determining the hippocampal representation of space, concerning which dimensions of information are task-relevant in a given task or condition. Changes in cognitive demands dramatically change the spatial map, as seen in various examples above, including, at the extreme, the Aronov et al. ${ }^{68}$ finding that when the continuous nonspatial and nontime task dimension of tonal frequency is determinative of behavior, hippocampal cells map tone frequency. In our own work, we have observed a range of combinations of rate and global coding in tasks that involve different memories in the same places ${ }^{56}$ and across places in the same environment ${ }^{69}$. Taken together, these observations have driven a growing interest in a broad capacity of mixed-selectivity neuronal responses for multiple relevant task dimensions as providing the mechanism by which cell assemblies of the kind found in the hippocampus can represent and associate complex concepts and cognitive maps.

\section{Nadel and Ranganath}

The hippocampus does not encode contexts in a vacuum - it encodes contexts in a real world that is populated with people and things ${ }^{2}$. Information that is stable in time and space, including object information ${ }^{70}$, is used to infer the current context and, by extension, the currently relevant hippocampal representation ${ }^{71}$. Conversely, the maps formed by the hippocampus include objects, specifying how they are related to one another in space and time ${ }^{72,73}$. In this sense, it is not a stretch to say that the reach of the hippocampus extends to literally any kind of information.

To make sense of what the hippocampus is doing, however, we need to distinguish between the dimensions of the current context and the people and things that are mapped to these dimensions. Activity patterns in the hippocampal network will be relatively similar across different objects that are co-localized in space or time, but hippocampal neurons will sharply distinguish between encounters with the same object in different contexts. To put it another way, the data in rats ${ }^{69,73,74}$ and in human functional MRI (fMRI) studies ${ }^{5,72,75}$ overwhelmingly show that spatial and temporal information are the most significant 
dimensions by which representations are organized in the hippocampus. Dimensions other than space and time can be encoded by the hippocampus ${ }^{68,76}$ if those dimensions are behaviorally relevant. However, information about time and space appears to be obligatorily encoded by the hippocampus even when it is not directly task-relevant. Thus, at least for the well-known subfields, location in space and time seems to have a special role ${ }^{2}$.

One caveat concerns subfield CA2 (ref. 77)—recent data seem to indicate that CA2 may be especially important for temporal ${ }^{78}$ and social ${ }^{79-81}$ memory. There is not nearly enough data on CA2, and it will be important to know the extent to which the representational scheme in CA2 parallels the more well-known subfields.

\section{Redish}

The evidence that some nonspatial information is encoded within hippocampal firing patterns is extremely well-supported by decades of work. But what this question is really asking (I think) is, how do hippocampal pyramidal cells encode nonspatial information?

There are three ways that nonspatial information is encoded within hippocampal firing patterns.

i. It can be encoded identically to place cell encoding, with cells creating a computational map across the range of values that the nonspatial information can take (Fig. 3). This does seem to occur for specific nonspatial factors such as time $^{82}$ and linear tones ${ }^{68}$. I will address this first possibility in my answer to the next question below.

ii. It can be encoded in a modulation of hippocampal firing, such that the spatial location of firing does not change, effectively providing a multiplicative gain to the place field. As long as the modulation is small relative to the place field itself, one can encode nonspatial information without disturbing spatial representations. The key is to differentiate this modulation phenomenon (which has been defined by the unfortunate term 'rate remapping') from the phenomenon of real remapping. (I would argue that the correct term for rate remapping is 'rate modulation' because the cells do not remap in rate remapping.) In a sense, the place field map forms a context in which nonspatial information is encoded through modulation.

iii. It can be encoded by a complete remapping of the place fields, as if the animal was treating the two situations as different environments. As has been well established, overlap between environments is essentially random (for example, ref. 83), suggesting that environments are represented as independent mappings. Models have shown that this remapping explanation provides good descriptions of subtask behaviors (such as directions on the linear track or during subtasks in an environment, in response to odor cues or other cues that differentiate goals). This remapping to differentiate environments develops over time and tends to occur when the nonspatial information informs the rat about different goal distributions within a space ${ }^{24}$. 


\section{Buzsáki}

No doubt, as it follows from the macroscopic anatomical connections of the hippocampus (Fig. 1).

\section{What is the function of time cells?}

Under conditions in which a rodent is stationary, time cells fire at a fixed times during a task (Fig. 3).

\section{Eichenbaum}

As introduced above, Tulving originally defined episodic memory as memory for events in space and time. With the accumulation of evidence that the hippocampus is essential for episodic memory, it should be fully expected that representations of space and time are both valuable to episodic memory. In particular, time cells may play a role in episodic memory by tagging when events occur in time, just as place cells map where events occur in space. So, the hypothesis is that time cells do for memory the same things that place cells do. Considered together, time cells and place cells organize events in different relational dimensions to provide cognitive maps in time and space, respectively.

With regard to movement, the question reflects a misunderstanding of how time and place cells are identified. The activity of time cells is not limited to just those conditions in which the animal is stationary. Time cells are typically studied during periods that are void of varying locations and events, to permit the observed neuronal firing to be unambiguously linked to elapsed time. It is hugely important to acknowledge that pure place cells-cells controlled only by location and not by time or events-are identified in situations that involve having the animal move in what can be considered random walks, that is, with no fixed or obligatory temporal ordering to their visiting of different places while they are engaged in foraging, thereby making it possible to unambiguously link neuronal firing to spatial position. By contrast, when movement trajectory is fixed, introducing a regular temporal ordering of locations in track running or fixed paths within an open field (as in work by Etan Markus), place cells do not only encode space but also encode direction, which reflects ordering in time. Using a fully parallel rationale, time cells are identified when position and behavior are held constant, for example, during treadmill running, thereby removing spatial and behavioral variables ${ }^{84}$. This does not mean that time is only encoded during immobility any more than place is encoded only when animals are moving randomly. Rather, place is coded during stereotyped movement, in which case it is strongly influenced by orderly direction of movement (i.e., track running), while time coding is strongly influenced by distance run on a treadmill when treadmill speed is varied ${ }^{84}$. My working hypothesis is that time cells and place cells automatically map continuous dimensions of experience and provide complementary frameworks for organizing relational and episodic memories, as seen in single neurons in animals as well as in human imaging (see publications by Charan Ranganath). Other variables become prominent for mapping experiences when time and space are removed as consistent variables and, instead, other consistent variables guide learning, as reflected both in neuronal firing patterns and in fMRI (see publications by Mayank Mehta as well as refs. 4,68,76). 


\section{Nadel and Ranganath}

There is little doubt that the hippocampus represents the temporal order in which the elements of an episode unfold ${ }^{2,85}$. It is interesting to note that the existence of time cells was predicted by computational models of hippocampal sequence encoding ${ }^{86,87}$. Also, time cells, like place cells, are context-specific, in the sense that changes in the behavioral context lead to changes in time fields ${ }^{9,10}$. This means individual time cells only carry information about moments of time within the context of a particular episode, just as individual place cells carry information about specific locations within specific spatial contexts. Putting it all together, our best guess is that time cells tell us about the relative order of events within an episode, just as place cells encode topological relationships in a spatial environment. What this means is that time cells in and of themselves don't provide information about absolute time (such as "this event happened last week"); they only provide information about time relative to an episodic context (such as, "this event happened after lunch arrived"). Again there is a parallel with place cells, which don't provide information about global position (i.e., it's not a GPS) but instead provide information about spatial relationships within a context.

This brings us to one more important point: although we speak of time cells and place cells, the terminology gives the false impression that hippocampal neurons are simple onedimensional feature detectors. In fact, most hippocampal cells appear to be tuned to both spatial and temporal information ${ }^{84}$, and many cells also show significant impacts of task context and goals ${ }^{69}$. It's also important to note that there is no simple isomorphism between neural coding and one's subjective sense of space and time. For instance, because sequence information can be a useful spatial cue, time cells probably contribute to spatial orientation, particularly when local cues are absent ${ }^{88}$.

\section{Redish}

I don't think time cells are a special type of cell. Instead, I think time cells are reflecting hippocampal computations in a nonspatial context that was first understood spatially. We know that place cells and time cells are the same cells and that they remap between the two representations. New experiments ${ }^{68}$ are now finding that nonspatial signals can be divided into place cell-like components (Fig. 3c). I suspect the key is continuous data versus dichotomous data. Time and space tend to be continuous, while episodic (nonspatial) events tend to be punctate and discrete. Importantly, the division of time by time cells in hippocampus depends on having a goal: when rats simply run on a running wheel in a place field, cells fire at their place fields forever at a specific phase, but when rats have a goal of running in the wheel for a set time, the ensemble divides up the time with time cells ${ }^{21,89}$. Division of time by time cells seems to depend on having a target time but does not depend on maintaining working memory across that time ${ }^{90}$, consistent with place cells that divide up space, whether that space is necessary for navigation or not (in contrast, for example, to striatal cells that only represent space, time, or other information when it provides useful signals for getting reward).

One thing that is very interesting is that phase precession in the absence of spatial changes depends on septal inputs, while phase precession during spatial traversal does not but the 
development of place fields in a novel environment does ${ }^{88}$. (This relates closely to my answer to the next question below that once you have developed external associations, those associations can drive sequences, but the initial sequences depend on self-motion information that depends on septal inputs for some aspect of the computation.)

\section{Buzsáki}

The concepts of space and time are our fundamental organizers of ideas. Their appeal goes back to the Newtonian framework, in which events take place in a large 'theater' or 'container' and unfold on a timeline. But in modern physics, there is no such container and no such timeline. The practically relevant and measurable variants of the Space and Time concepts are distance and duration, whose units are quantified by human-made instruments, such as rulers and clocks. In the laboratory, we compare the evolution and the rate of change of neuronal firing patterns and cell assembly sequences against the units of such instruments and often find reliable correlations.

However, the correlation between neuronal activity and distance or duration is perhaps not so important. The critical question is how downstream targets of hippocampal neurons interpret those patterns. Are there separate downstream classifiers that interpret hippocampal output spikes as distance or duration? That does not seem to be the case, so it may well be that the brain does not interpret them as separate entities. Instead, distances between place fields are represented as proportional durations within hippocampal theta oscillations ${ }^{91,92}$. Distance (or displacement) and duration are related to each other via instantaneous speed (or velocity). The velocity signal can warp the duration of the theta cycle, so that the relationship between the animal's position or its goals and the theta phase of pyramidal cell spikes remains invariant ${ }^{93,94}$.

Importantly, even if the evolution of neuronal activity is reliably correlated with the temporal succession of events ('timing of representations'), such correlation does not mean that neuronal activity computes time ('representation of time') ${ }^{95,96}$. One may precisely track distance or duration from the activity of various regions in the brain and even from the spinal cord, though it is unlikely that our mental percept of time and space is created separately in each brain region. Of course, distance and duration will continue to be useful for comparative measures and discoveries in neuroscience. However, despite the convenience of these measures, we must resist jumping to the conclusion that the rate of change of neuronal assemblies amounts to the measurement or creation of space or time in either humans or other animals ${ }^{96}$.

\section{What is the role of the grid cells in the cognitive map?}

The excitement about grid cells comes from the fact that we now have reasonable computational models of how the grid cell network of the medial entorhinal cortex could serve as a two-dimensional analog integrator of velocity ${ }^{97}$. Thus, if information about actual self-motion signals (speed and direction) is put into the integrator, the activity bumps simulate the actual movement of the animal, and this can perform the function of path integration (knowing where you are based on a past position and the integration of your intervening velocity). Such path integration (Fig. 4) can also be used during imagined 
movement ${ }^{98}$, i.e., if I move with this speed and direction, what positions will I come to? This is a cognitively important operation; if the computed movement of the activity bumps intersects a reward site, that's a good direction to go in. But how does data bear on the idea of integration? Now that it has been shown that grid cell firing patterns are strongly determined by environmental cues ${ }^{99}$ and, conversely, that they fall apart in the dark ${ }^{100}$, is it still appropriate to talk about grid cell firing patterns as driven by path integration?

\section{Eichenbaum}

We see grid cells integrate on the treadmill to produce a signal of distance traveled and elapsed time. What other empirical evidence supports the idea that grid cells integrate? Early work (Hafting et al., 2005) showed that grid cell firing patterns persist during open-field foraging in the dark, suggesting that somewhere in the brain, information generated by selfmotion is encoded. But the information about location in the dark could originate in structures afferent to the grid cells, including the hippocampus, or could arise from frequent interactions with environmental boundaries (not well controlled in ref. 101). In addition, the new evidence showing that grid cell firing patterns are strongly determined by environmental cues ${ }^{99}$ and, conversely, that the grid pattern falls apart in the dark ${ }^{100}$, indicates that grid cells are not driven solely by self-generated cues and that self-generated cues are not sufficient to sustain grid cells. Some have argued that the integrator of self-motion cues needs to be occasionally reset because of error accumulation, and indeed, path integration is effective for surprisingly brief journeys in animals and humans (see publications by Larry Squire). In the real world, it is well known that path integration is very poor as a strategy for navigation (see work by John Huth and work from my own lab). Furthermore, while the Nobel prizewinning studies that focus on open-field foraging have inspired many to attribute a dedicated role for the medial entorhinal cortex (MEC) and grid cells in navigation and path integration, MEC is essential to spatial and nonspatial memory that does not involve navigation or path integration (see publications from Magdalena Sauvage), and grid cells also code time and tone frequency when these are prominent variables during learned behavior. Also, in humans, a reflection of the grid pattern in the fMRI signal occurs when subjects identify events organized by arbitrary, nonspatial stimulus dimensions (neck length and leg length of stick birds ${ }^{102}$ ). So, grid patterns can be generated by externally generated nonspatial cues. While the grid pattern is unique as contrasted with the typical property of single receptive fields in neurons of the brain, the role of this form of representation seems best related to a coding of spatial, temporal, and abstract context as pervasive, continuous dimensions that characterize contexts of our everyday experience.

\section{Buzsáki}

The discovery of the grid cells ${ }^{101}$ was among the most spectacular moments in neuroscience. Initially, it appeared that mapping and navigational functions, attributed to the hippocampus, reside in the entorhinal cortex, freeing up the hippocampus for memory processing. The pendulum has swung back and forth a few times over the past decade, intensifying the debate, forcing us to think in a larger context and recruiting many new investigators into the field. It is now becoming clearer that functions do not reside in this or that structure but emerge through their interactions. The hippocampus-entorhinal cortex- 
prefrontal cortex partnership is involved in almost everything that has been traditionally attributed to one member only under a particular testing condition.

However, maps are static, whereas navigation — whether in physical or cognitive space ${ }^{103}$ is dynamic. Without motion, there is no grounding; distances and durations cannot be calibrated, and therefore neuronal patterns cannot acquire meaning. Motion is sensed by velocity detectors. During real-world navigation, the rate of change within the assembly sequences is controlled by the animal's velocity. In brains of increasing complexity, many environmentally driven functions become internalized ${ }^{104}$ and the rate of change-for example, during memory recall, imagination, or planning-may become under the control of a hypothetical mechanism known as 'attention', in lieu of velocity. In a thinking brain, therefore, attention may be the substitute for velocity, which can maintain the grid dynamics in the absence of locomotion. This speculation is supported by the presence of hexagonal grid-like dynamics organizing both spatial and non-spatial conceptual representations in a variety of higher-order cortical regions without overt motion. Grid dynamics can, therefore, also support abstract knowledge ${ }^{102,105}$, not only maps.

\section{Nadel and Ragananth}

By focusing on the response properties of grid cells and reducing the complexity of the entorhinal-hippocampal circuit to a simple visuomotor integration problem, the challenges in modeling this circuit become tractable. This approach is problematic, though, because grid cells provide a relatively narrow window of insight into the hippocampal formation.

Grid cells are a small subpopulation of cells in the superficial layers of the medial entorhinal cortex. In addition to grid cells, there are border and head direction cells in MEC, and collectively, these comprise only a fraction of the principal cell population in MEC. Recent work has shown that the remaining two-thirds of the MEC cell population is also spatially selective, despite the fact that the tuning of these cells is not as obvious as is the tuning for the known cell types. It is likely that grid cells play a limited role in spatial representation in the hippocampus, though they might play an important role in temporal processing ${ }^{106-108}$. Diehl et al. ${ }^{109}$ reveal that the nongrid population, on the other hand, may be quite interesting -these cells, like CA1 place cells, show global remapping (i.e., a change in the corresponding spatial context representation) if sensory cues do not match previously known environments. This work nicely complements findings from Keene et al. ${ }^{110}$ showing that MEC neurons carry considerable information about time, spatial context, spatial location, and the locations of objects in an environment.

To cut to the chase, research on grid cells has conveyed the impression that the MEC has an impoverished representation of space, but recent research shows quite the opposite. MEC may have a much richer representation of events than previously thought, with many of the necessary ingredients of a cognitive map (see Hardcastle et al. ${ }^{111}$, this issue, for a similar perspective).

\section{Redish}

When discussing self-motion, we need to remember two important issues. First, dead reckoning (path integration) depends on self-motion cues, and thus, it is first important to 
determine what information is being used to measure the dead-reckoning distances. There are lots of useful signals that can provide self-motion information (proprioception, visual flow, etc.). In other animals, specific signals are used; when those signals are disrupted, the distance measurements are disrupted. (For example, desert ants count steps ${ }^{112}$ and goslings integrate visual flow ${ }^{113}$.) Grid cells could fall apart if the cues that rats use are disrupted (such as a lack of visual flow in the dark).

Second, because self-motion cues only provide information about changes in coordinates, errors grow with distance and time. Thus it could be possible that the grid cell representation could be intact, but because it is drifting relative to the external world, we can't see the grid relationship. (This is related to Buzsáki's readout point: we are missing the readout because the rat thinks it is somewhere else and we are unable to see that. Figure 3 of Chen et al. ${ }^{100}$ suggests that this might be the case. Figure 4 of the same paper suggests that the integration is good for a few seconds but starts to fall apart within a minute or so.)

The elevator-task data from the McNaughton lab in the 1990s examined how external cues (visual, tactile, or olfactory) could provide reset information to correct for errors in selfmotion cues ${ }^{6,114,115}$. These experiments supported the update-and-reset models, which suggested that the hippocampal role was to correct for errors in self-motion cues through learned associations between external "where am I" information and extra hippocampally represented coordinate systems ${ }^{24,116,117}$. The key question, however, is how far an animal can travel before errors in the self-motion information build up too much to be useful. The Chen et al. paper ${ }^{100}$ suggests that this distance may be quite short. An interesting question is whether the determinant of that reset depends on distance traveled or time since the last reset. Data from Redish et al. ${ }^{114}$ suggest that reset from incompatible environmental cues (when the environmental information does not match the dead-reckoning information) is dependent on the time of exposure to the incompatibility, not the distance traveled. Data from Gothard et al. ${ }^{115}$ suggest that this reset depends on the amount of information being provided to the animal (it took longer to reset in the dark).

A further clue to this reset phenomenon is in rodent exploration behavior in novel environments. In novel environments, rodents define a home base, and then make excursions out of that home base, proceeding slowly, until after a certain distance (or time?) they flee back to the home base in a very straight line ${ }^{118}$. Models suggest that these excursions are associating external cues with coordinates until those coordinates become unreliable and the animal has to return to the home base to reset the coordinate system ${ }^{24}$. Over time, these excursions increase in distance and time until the environment is well-covered (and presumably familiar). These initial distances are very short, which may suggest that we shouldn't be considering this as the same level of dead-reckoning that is done by desert ants or homing pigeons (both of which do use external cues for additional velocity and direction information $)^{53}$. Nevertheless, the time before grid cell breakdown in ref. 100 is similar to that of the reset phenomena seen in the McNaughton elevator task papers ${ }^{114,115}$ and to that of the exploration excursions seen in ref. 118, suggesting that self-motion information is still the key to understanding grid-cell behavior. 


\section{Can the concept of a map be generalized as a fundamental cognitive strategy?}

Is the representation of time an artifact of moving through places in a particular order, or does the hippocampus create a temporal framework (map) as it might seem to do for space? Are hippocampal representations of both time and space really just prominent examples of a fundamental code for mapping adjacencies in continuous dimensions of experience? In light of recent reports on hippocampal and entorhinal mapping of multiple nonspatial dimensions (time, social space, and abstract associative spaces), as well as multiple spatial dimensions (direction, distance, speed), isn't it more appropriate to refer to Tolman's original view that the cognitive map is a mapping of dimensions in cognitive space, not solely physical space?

\section{Eichenbaum}

Several studies have observed entorhinal time cell sequences when the animal's location is constant, while running in a wheel or on a treadmill or when the head is fixed; therefore, time cells are not an artifact of moving through places in order. Instead, in diverse tasks hippocampal cells fire at adjacent positions along a broad range of continuous dimensions of experience (physical space, time, tonal frequency). In addition, multiple studies have shown that the hippocampus is essential for the construction of conceptual spaces in rats, including stimulus hierarchies (Dusek \& Eichenbaum, 1997) and associative networks (Bunsey \& Eichenbaum, 1996). Correspondingly, fMRI studies have described hippocampal and entorhinal mapping of associative, social, and abstract spaces in humans (Milivojevic \& Doeller, 2013; Schiller et al., 2015; Schlicting et al., 2017; Gavert et al., 2017). These findings suggest that hippocampal memory organization may extend to the mapping of relationships between events in any cognitive space. These findings make it increasingly difficult to claim a special role for physical space per se, beyond its presence as a salient dimension that organizes experiences, as commonly emphasized in studies of place cells. It is highly gratifying that this perspective, which began many years ago as the notion of relational representation, is finding extensive empirical support from studies on neuronal firing patterns in rodents, in functional imaging in humans, and across species in a wide range of relational memory for different types of spatial representations (route and survey mapping) and for many types of nonspatial continuous and discontinuous dimensions that compose the 'spaces' of cognitive maps.

\section{Nadel and Ranganath}

Our understanding of hippocampal processing of space and time can probably apply to any relevant dimension of experience, but that does not mean that the hippocampus encodes any and all dimensions indiscriminately. Space and time play a central role in hippocampal function.

One way to consider this question is through an evolutionary lens: did the hippocampus evolve as a domain-specific organ to solve spatial location problems, or did it evolve as a domain-general organ to solve the abstract problem of mapping continuous dimensions of any sort, as alluded to by the other commenters? While we can all agree that, by a certain point in evolution, the hippocampus can play this broader role, it is still relevant to ask what 
drove the evolutionary emergence of this brain system in the first place. We consulted an expert, Clark Barrett (UCLA), who we quote (with his permission): "At one level, the reasons brain variants are selected for is always concrete (e.g., live or die, reproduce or not) - but even below that, it seems that they can only have those payoffs if they help organisms do things that are concrete, at least in their immediate instantiation (e.g., find food, avoid predators, remember their way to a good foraging area, etc.)." It's not hard to see the competitive advantage afforded by a brain mechanism that organizes experiences according to spatial and temporal relationships. The functions that we've outlined for the hippocampus would not only specify the locations of foraging sites and potential predators but also enable context-dependent representations of these locations. In other words, the hippocampal map can support memory for the location of a tree that only has fruits in the summertime or the site of a water source that is frequented by predators at night but safe during the daytime.

There is no doubt machinery that evolved to index experience by spatial and temporal context can be flexibly recruited to map dimensions beyond space and time. The critical point, however, is that the hippocampus always indexes information about space and time; mapping of other dimensions depends on behavioral relevance. Learning that a variable is behaviorally relevant is, in turn, based on reference to a spatiotemporally indexed episode ${ }^{8}$.

\section{Redish}

We know that rats ${ }^{68}$ and humans ${ }^{119}$ can create cognitive map-like spaces in hippocampal representations. Furthermore, we know that, at least in humans, the effects of episodic future thinking on decision-making depend on hippocampus ${ }^{120,121}$. I think there are two ways that the concept of a cognitive map can be generalized, which are best understood in terms of how the maps are used.

In my view, one role is that of finding new connections through creativity and mindwandering. This is the role of the 'default mode network' (introspective processing through rumination about connections and processes) and is likely occurring during hippocampal replay (which we now know is not actually replaying so much as it is exploring ${ }^{122-124}$ ). I would expect that these connections can be made in more general spaces than physical space, but I don't know of any experiments that have tested the role of hippocampus in these more general 'insightful' self-discoveries.

The other role is as part of a more general planning system in which hippocampus is the key to mental time travel, allowing the construction of search plans through future outcomes. Whereas mind-wandering allows the discovery of new connections, presumably for longterm future use ("the library and the school are close together"), planning is about immediate goals ("I can get to the library by taking this shortcut."). For many years, it has been known that one can contrast map-based decisions (that allow shortcuts, new paths, etc., which are slow to execute but flexible in their execution) with route-based decisions (which are fast but inflexible). In my view, these map-based decisions are a subset of the deliberative planning process that depends on searching through the episodically constructed futures (via the cognitive map). Given the role of hippocampus in episodic future thinking in humans, it is likely that this is part of a more general process. 


\section{Buzsáki}

The cognitive map metaphor is another very useful organizational tool. The essence of a map is the graph relationship among its nodes. In a directed graph, it is possible to calculate the shortest path sequence from any node to any other node. The hippocampus, as the neuronal embodiment of Tolman's postulated cognitive map, was suggested to compute distances and angles in the environment, thereby permitting solutions to spatial problems ${ }^{2}$. The large recurrent excitatory system of hippocampal CA3 neurons may meet the requirements of such a graph, assuming that distances between landmarks (i.e., graph nodes) in the environment are encoded as synaptic strengths ${ }^{125}$. It is important to note that search algorithms for finding the optimal path are general and not specific to the spatial domain. For example, graph representations are routinely used for mapping synonyms and antonyms in language and to compute eye-centric, head-centric, and body-centric representations in the parietal cortex. All of these relationships can be conceptualized as distances, although they are not measured in metric units. Thus, Tolman's cognitive map idea can be generalized to nonspatial domains as well as long as we remember that it is only a metaphor. The thetaoscillation-dependent mechanisms of navigation generalize to memory-supporting mechanisms ${ }^{103}$. Therefore, referring to a set of relationships ${ }^{3}$ is perhaps more appropriate than the two-dimensional map metaphor.

\section{The hippocampus displays prominent oscillatory activity in the theta and gamma frequency ranges (Fig. 5); do these oscillatory activities have an essential role of the cognitive processes mediated by the hippocampus?}

If so, is this uniquely important for the hippocampus, or are the oscillations relatable to a more a general brain code?

\section{Buzsáki}

In general, segmentation or chunking of information is an important brain operation, irrespective of whether it is assisted by saccadic eye movements, echolocation, external stimuli, or, in their absence, by internally generated brain rhythms. In the hippocampus, theta oscillations provide a time-compressing mechanism that converts distances between place representations into proportional durations defined by phase offsets of the theta waves ${ }^{91,126}$. Theta mechanisms also appear essential for the generation of self-organized neuronal assembly sequences (Fig. 5), spatial navigation, and episodic memory ${ }^{88}$.

Furthermore, theta oscillations define the partners of the hippocampus throughout the limbic system and coordinate cell assemblies across these partner structures. While theta frequency varies as a function of behavior, at each moment in time the frequency is exactly the same in all these partner regions. This is how theta oscillations support inter-regional coordination.

However, theta oscillations do not synchronize neurons in the entire structure. Instead, theta waves travel from the septal to the ventral pole of the hippocampus, coordinating spiking activity and gamma power to the local theta phase ${ }^{127,128}$. The mechanisms that read out such spatially distributed, time-shifted neuronal information remain to be understood, but it is 
clear from these dynamics that downstream readers of hippocampal activity should take into account such spatially and temporally segmented content.

Gamma waves are higher-frequency oscillations that are coordinated by theta oscillations. Gamma waves contain cell assemblies that are concatenated into assembly sequences ('neural words') through cross-frequency phase-coupling between theta and gamma (Fig. $5)^{129,130}$. Since theta oscillations also coordinate inter regional communications, this crossfrequency theta-gamma coupling can provide a readout of afferent drive. By placing electrodes in different hippocampal layers along the pyramidal cell dendrites, one can quantify the instantaneously changing magnitudes of the upstream activities. For example, in area $\mathrm{CA} 1$, the power of low-frequency gamma $(30-60 \mathrm{~Hz})$ on the descending phase of theta in the stratum radiatum varies as a function of the strength of the CA3 inputs, whereas the power of mid-frequency gamma $(60-110 \mathrm{~Hz})$ in stratum lacunosum moleculare, around the peak of theta waves, varies as a function of the magnitude of the entorhinal input. The relative strengths of these upstream inputs predict the theta phase of spikes during learning, REM sleep, retrieval of memories, and traversing the place fields (Fig. 5; and see refs. 131133).

Oscillations in different regions share many physiological benefits, such as providing temporary gains in excitatory drive while balancing excitation and inhibition and concatenating cell assemblies. However, it must be noted that no rhythm has a prescribed cognitive function by virtue of its physiological properties. Instead, the computational benefits and behavioral correlates of any given oscillation will depend on the substrate from which that rhythm emanates ${ }^{134}$.

\section{Eichenbaum}

Considerable recent evidence on prefrontal-hippocampal interactions supports the idea that synchronized oscillatory activity in the gamma and theta ranges reflect coordinated communication within local networks and across connected brain areas, respectively. Our work should focus on identifying the information communicated in these oscillations. For example, with regard to long-range communication, the synchronization of theta oscillations between the prefrontal cortex and hippocampus reflects a bidirectional communication that supports the control of memory organization ${ }^{135}$.

\section{Nadel and Ranganath}

Before answering this question, it is important to clarify that theta oscillations are not specific to the hippocampus. In fact, theta oscillations have been known to occur in human EEG since the recordings of William Grey Walter in the mid-1940s. The theta rhythm was so named by Grey Walter to apply to oscillations in the human neocortex, and the moniker was only later applied to oscillations in the rodent hippocampus. Scalp-recorded theta oscillations are most likely volume-conducted from multiple neocortical sources, including medial pre-frontal cortex. Theta oscillations have also been recorded in the striatum in humans and rats ${ }^{136}$. In other words, it's well known that theta oscillations occur outside of the hippocampus, but it's not yet clear whether or how these various theta oscillations are related to one another. Even if we assume that hippocampal theta is closely related to 
extrahippocampal theta, it is probably a mistake to think that we will arrive at a single cognitive function for theta oscillations. For instance, in humans, theta activity is strongly related to episodic memory retrieval, but it is also related to working memory maintenance and error processing (see ref. 137 for review). Instead, we might want to focus on theta as a general brain mechanism for synchronizing ordered spike-timing amongst large neural ensembles ${ }^{129}$. As such, theta may be a key mechanism for inter-regional communication. For instance, if contextual information is relevant for an upcoming decision, hippocampalprefrontal theta synchrony can prioritize hippocampally modulated prefrontal neurons to guide action ${ }^{135,138}$.

\section{Redish}

Absolutely. The oscillatory processes in hippocampus are a critical part of its computational processing. The key is the sequences formed by cell spiking within each theta cycle (the 'theta sequence'). It is very clear that theta sequences are real, that they reflect important information within the cognitive system (potential paths and goals), and that they can change on a cycle-by-cycle basis based on immediate goals ${ }^{94,139}$. Of course, two important unanswered questions are (i) whether the sequences are somehow modified by the theta cycle or whether the theta cycle merely reflects some regular sequence reset and (ii) what the relationship is between sequences during theta and sequences during sharp waves.

Whether the coupling to gamma has an explicit role in cognition or whether it is just an epiphenomenon of how the neural pro cessing works mechanistically is less clear, but it is very clear at this point that there are multiple gamma rhythms (gamma, gam-ma $a_{M}$, gamma $_{H}$ ) that reflect processing from different components of the system (within CA1: gamma $_{L}$ reflecting CA3 drive, gamma ${ }_{M}$ reflecting entorhinal, and gamma ${ }_{H}$ reflecting intraCA1 processes) and occur at different times through the theta cycle. These rhythms reflect different parts of the sequence; changes in the sequence (for example, sequence length) occur with changes in relative strengths of the components of these gamma rhythms.

\section{Lisman}

In providing an overview of hippocampal function, it may be useful to discuss each of the terms in the acronym for a hippocampal model: the SOCRATIC model (sequences of condensed representations, autocorrected and time-compressed in context) ${ }^{140}$.

We know that individual items can be recognized without the hippocampus but that sequences cannot. This meshes with the concept of episodic memory, which often involves sequences of events. The discovery that place cells with sequential position along a path are active at sequential phases of individual theta cycles ${ }^{126}$ provided clear physiological evidence for a specialized process that deals with sequences. The interplay of theta and gamma oscillations organizes a temporal code appropriate for multi-item sequences (ratecoding cannot describe hippocampal function). The role of oscillations is now accepted in the hippocampus and will hopefully inspire investigators in other fields who seem skeptical about the importance of brain oscillations.

Sequential positions during a theta cycle are represented during sequential gamma cycles, cycles that occur about $25 \mathrm{~ms}$ apart. However, these positions are actually visited by the rat 
over the much longer time period of a second; it is this difference that is termed 'time compression ${ }^{91}$. This time-compressed readout of sequence information appears to be a form of mind travel (mind wandering), driven by integration of a nonreal velocity vector that is fed into the grid cell network, which then drives the apparent position represented by the active hippocampal place cells to successive positions in the direction of the velocity vector (usually straight ahead of the animal; Fig. 4). I (J.L.) suspect that this process mediates the most important cognitive advantage of this system: for example, it allows the rat to quickly determine whether there is a cat associated with any of the positions that might be reached if the cat goes through a hole in a wall. If one accepts the role of grid cell networks as a twodimensional integrator, then it seems natural to consider that similar networks could be used to navigate within other cognitive dimensions. Thus, for instance, beauty might be one dimension in a two-dimensional grid-cell network map, and one could envision an artificial beauty vector that produced exploration of 'beauty space' as a scalar value of beauty was system atically varied. Thus, one interpretation of the low fraction of spatial grid cells in the medial entorhinal cortex is that one or more of the dimensions represented by other 'grid cells' is a nonspatial dimension.

The term 'autocorrected' refers to the special attractor properties of the CA3 network. This network has extensive recurrent excitatory connections that allow pyramidal cells to excite each other. Such networks have been a central focus of the theoretical analysis of memory networks. On the basis of this theoretical work and complementary experiments, it appears that $\mathrm{CA} 3$ is one of the clearest examples of an autoassociative network and performs the important functions of pattern completion (one part of a memory recalls another) and error correction (as required so long sequences can be correctly recalled) ${ }^{142-144}$.

The above discussion dealt extensively with the role of space and time as a description of the representations carried by hippocampal cells. The late Howard Eichenbaum deserves special credit for his efforts to move the field to a conceptual framework capable of incorporating the now-extensive experimental data for nonspatial representations. I (J.L.) would like to introduce one additional perspective. A remarkable anatomical feature of the input to the hippocampus is the dual inputs it receives: one from the medial entorhinal cortex and one from the lateral entorhinal cortex. We still don't know how to put functional labels on these inputs; a what-versus-where dichotomy is popular, but I (J.L.) favor a dichotomy in which the medial entorhinal represents properties of self, whereas the lateral entorhinal represents properties of the external world (nonself ${ }^{141}$ ). Perhaps very-high-resolution fMRI will be able to resolve the basis of this dichotomy in humans. The other major determinant of representation is context. A justice of the US Supreme Court said that he couldn't define pornography but he knew it when he saw it. Context seems similarly difficult to define, though behaviorists have developed indirect assays. Now it seems that electrophysiology opens the door to a measurement-based approach with a clear definition: a new context is one that is sufficient to evoked global remapping (Fig. 2). On the other hand, simply inserting an object into a known context will link a position with the representation of that object by rate remapping. The need to obtain a firm mechanistic understanding of rate remapping cannot be underestimated: this is what makes the map (a two-dimensional spatial map) provided by the grid cells into an annotated two-dimensional map, i.e., a cognitive map. Focusing on the annotation problem, the use of rate remapping to associate a sensory 
stimulus with a place can be implemented plausibly, but what if there are multiple associates with a place? Perhaps time cells create an extra dimension that can solve this problem; computational work is needed to assess this possibility. These considerations hopefully give some concreteness to the idea that the hippocampal region may use "condensed representations" in which cell firing depends on position, other cognitive dimensions and sensory stimuli.

The process of understanding memory-guided behavior will necessarily require new ideas about large-scale integration. The hippocampus is a particular kind of memory store that can interact with the goal-directed (nonhabit) action choice system to determine what action should be taken in a given context, but there are other memory stores, including a wellcharacterized habit-memory store in the basal ganglia. We can now watch individual memory replay events in the hippocampus during behavioral choice, and the slowness of the process is notable; individual episodes are replayed in succession. In contrast, the habit system, because it stores only a statistical average over episodes, can access that variable quickly in a single pass. Still, in a world where one needs to make a complex decision with limited experience, playing out options and deciding between them may be more advantageous than averaging over experiences. For instance, having had a single near-death experience with a cat, a mouse might favor not going through a particular hole, even if the probability of cat was low. Understanding the cognitive rules that determine the utilization of different memory stores by downstream action-selection networks is now a feasible goal that will provide understanding of how the hippocampus functions within the large scale system that determines behavior.

\section{Acknowledgments}

The editors are extremely grateful to Dr. Karen Shedlack (Howard Eichenbaum's widow), Denise Parisi (Assistant Director, Center for Memory and Brain at Boston University), and Dr. Neal Cohen (Department of Psychology at University of Illinois) for their efforts in ensuring that Dr. Eichenbaum's final revisions were located, revised, and included in this publication. J.L. was supported by NIH grant U19NS104590. C.R. was supported by a Vanneavar Bush Faculty Fellowship (Office of Naval Research Grant N00014-15-1-0033). Any opinions, findings, and conclusions or recommendations expressed in this material are those of the author(s) and do not necessarily reflect the views of the Office of Naval Research or the U.S. Department of Defense. H.E. was supported by NIH/NIMH grants R01 MH051570, R01 MH095297 and R01 MH052090. A.D.R. was supported by NIH grants MH080318 and MH112688. G.B. was supported by the Simons Foundation and by NIH grants MH54671, NS 090583, NS090526 and NS074015.

\section{References}

1. Tulving, E., Donaldson, W., Bower, GH. Organization of Memory. Academic Press; 1972.

2. O'Keefe, J., Nadel, L. The Hippocampus as a Cognitive Map. Clarendon Press; Oxford University Press; 1978.

3. Cohen, NJ., Eichenbaum, H. Memory, Amnesia, and the Hippocampal System. MIT Press; 1993.

4. Deuker L, Bellmund JL, Navarro Schröder T, Doeller CF. eLife. 2016; 5:e16534. [PubMed: 27710766]

5. Nielson DM, Smith TA, Sreekumar V, Dennis S, Sederberg PB. Proc Natl Acad Sci USA. 2015; 112:11078-11083. [PubMed: 26283350]

6. Gothard KM, Skaggs WE, Moore KM, McNaughton BL. J Neurosci. 1996; 16:823-835. [PubMed: 8551362]

7. Muller RU, Kubie JL. J Neurosci. 1987; 7:1951-1968. [PubMed: 3612226]

8. Ekstrom, AD., Ranganath, C. Hippocampus. 2017. https://dx.doi.org/10.1002/hipo.22750 
9. MacDonald CJ, Lepage KQ, Eden UT, Eichenbaum H. Neuron. 2011; 71:737-749. [PubMed: 21867888]

10. MacDonald CJ, Carrow S, Place R, Eichenbaum H. J Neurosci. 2013; 33:14607-14616. [PubMed: 24005311]

11. Ferbinteanu J, Shapiro ML. Neuron. 2003; 40:1227-1239. [PubMed: 14687555]

12. McNaughton BL, Barnes CA, O'Keefe J. Exp Brain Res. 1983; 52:41-49. [PubMed: 6628596]

13. Wood ER, Dudchenko PA, Eichenbaum H. Nature. 1999; 397:613-616. [PubMed: 10050854]

14. Lee JLC, Nader K, Schiller D. Trends Cogn Sci. 2017; 21:531-545. [PubMed: 28495311]

15. Dongaonkar B, Hupbach A, Gomez R, Nadel L. Psychopharmacology (Berl). 2013; 226:769-779. [PubMed: 23404063]

16. Hupbach A, Gomez R, Nadel L. Memory. 2009; 17:502-510. [PubMed: 19468955]

17. Hupbach A, Gomez R, Nadel L. Psychon Bull Rev. 2011; 18:787-797. [PubMed: 21647786]

18. Hupbach A, Hardt O, Gomez R, Nadel L. Learn Mem. 2008; 15:574-579. [PubMed: 18685148]

19. Schlichting ML, Preston AR. Curr Opin Behav Sci. 2015; 1:1-8. [PubMed: 25750931]

20. Lisman JE. Neuron. 1999; 22:233-242. [PubMed: 10069330]

21. Pastalkova E, Itskov V, Amarasingham A, Buzsáki G. Science. 2008; 321:1322-1327. [PubMed: 18772431]

22. Dede AJO, Frascino JC, Wixted JT, Squire LR. Proc Natl Acad Sci USA. 2016; 113:13480-13485. [PubMed: 27821761]

23. Tolman EC. Psychol Rev. 1948; 55:189-208. [PubMed: 18870876]

24. Redish, AD. Beyond the Cognitive Map: from Place Cells to Episodic Memory. MIT Press; 1999.

25. Schmitzer-Torbert NC, Redish AD. Neuroscience. 2008; 153:349-360. [PubMed: 18406064]

26. Berke JD, Breck JT, Eichenbaum H. J Neurophysiol. 2009; 101:1575-1587. [PubMed: 19144741]

27. Jung MW, Qin Y, McNaughton BL, Barnes CA. Cereb Cortex. 1998; 8:437-450. [PubMed: 9722087]

28. Powell NJ, Redish AD. Front Behav Neurosci. 2014; 8:120. [PubMed: 24795579]

29. Hartley T, Lever C, Burgess N, O'Keefe J. Phil Trans R Soc Lond B. 2013; 369:20120510. [PubMed: 24366125]

30. Brown TI, et al. Science. 2016; 352:1323-1326. [PubMed: 27284194]

31. Viard A, Doeller CF, Hartley T, Bird CM, Burgess N. J Neurosci. 2011; 31:4613-4621. [PubMed: 21430161]

32. Hannula DE, et al. Front Hum Neurosci. 2010; 4:166. [PubMed: 21151363]

33. Voss JL, Bridge DJ, Cohen NJ, Walker JA. Trends Cogn Sci. 2017; 21:577-588. [PubMed: 28625353]

34. Hannula DE, Ranganath C. Neuron. 2009; 63:592-599. [PubMed: 19755103]

35. Jutras MJ, Buffalo EA. Proc Natl Acad Sci USA. 2010; 107:401-406. [PubMed: 20018683]

36. Liu ZX, Shen K, Olsen RK, Ryan JD. J Neurosci. 2017; 37:599-609. [PubMed: 28100742]

37. Hannula DE, Ryan JD, Tranel D, Cohen NJ. J Cogn Neurosci. 2007; 19:1690-1705. [PubMed: 17854282]

38. Ryan JD, Althoff RR, Whitlow S, Cohen NJ. Psychol Sci. 2000; 11:454-461. [PubMed: 11202489]

39. Aly M, Ranganath C, Yonelinas AP. Neuron. 2013; 78:1127-1137. [PubMed: 23791201]

40. Lee ACH, Barense MD, Graham KSQ. J Exp Psychol B. 2005; 58:300-325.

41. Kravitz DJ, Saleem KS, Baker CI, Mishkin M. Nat Rev Neurosci. 2011; 12:217-230. [PubMed: 21415848]

42. Nadel L, Peterson MA. J Exp Psychol Gen. 2013; 142:1242-1254. [PubMed: 23895347]

43. Ranganath C, Ritchey M. Nat Rev Neurosci. 2012; 13:713-726. [PubMed: 22992647]

44. Aggleton, JP. Proc R Soc Lond B. 2014. https://dx.doi.org/10.1098/rspb.2014.0565

45. Bohbot VD, Allen JJ, Nadel L. Ann NY Acad Sci. 2000; 911:355-368. [PubMed: 10911885]

46. Bohbot VD, et al. Neuropsychologia. 1998; 36:1217-1238. [PubMed: 9842767]

47. Maguire EA, Nannery R, Spiers HJ. Brain. 2006; 129:2894-2907. [PubMed: 17071921] 
48. van der Meer M, Kurth-Nelson Z, Redish AD. The Neuroscientist. 2012; 18:342-359. [PubMed: 22492194]

49. Rosenzweig ES, Redish AD, McNaughton BL, Barnes CA. Nat Neurosci. 2003; 6:609-615. [PubMed: 12717437]

50. Kelemen E, Fenton AA. Neurobiol Learn Mem. 2016; 129:50-59. [PubMed: 26748023]

51. Jadhav SP, Kemere C, German PW, Frank LM. Science. 2012; 336:1454-1458. [PubMed: 22555434]

52. de Lavilléon G, Lacroix MM, Rondi-Reig L, Benchenane K. Nat Neurosci. 2015; 18:493-495. [PubMed: 25751533]

53. Gallistel, CR. The Organization of Learning. MIT Press; 1993.

54. Eichenbaum H. J Neurophysiol. 2017; 117:1785-1796. [PubMed: 28148640]

55. Johnson A, Redish AD. J Neurosci. 2007; 27:12176-12189. [PubMed: 17989284]

56. Wood ER, Dudchenko PA, Robitsek RJ, Eichenbaum H. Neuron. 2000; 27:623-633. [PubMed: 11055443]

57. Frank LM, Brown EN, Wilson M. Neuron. 2000; 27:169-178. [PubMed: 10939340]

58. Huth, JE. The Lost Art of Finding Our Way. Belknap Press, Harvard University Press; 2013.

59. Spiers HJ, Maguire EA. Hippocampus. 2007; 17:618-626. [PubMed: 17492693]

60. Jeffery KJ, Gilbert A, Burton S, Strudwick A. Hippocampus. 2003; 13:175-189. [PubMed: 12699326]

61. Robbe D, Buzsáki G. J Neurosci. 2009; 29:12597-12605. [PubMed: 19812334]

62. Ramirez S, et al. Science. 2013; 341:387-391. [PubMed: 23888038]

63. Redondo RL, et al. Nature. 2014; 513:426-430. [PubMed: 25162525]

64. Kinsbourne, M., Wood, F. Short-term memory pro cesses and the amnesic syndrome. In: Deutsch, JA., editor. Short-Term Memory. Academic Press; 1975. p. 257-291.

65. Vargha-Khadem F, et al. Science. 1997; 277:376-380. [PubMed: 9219696]

66. Leutgeb JK, et al. Neuron. 2005; 48:345-358. [PubMed: 16242413]

67. Wills TJ, Lever C, Cacucci F, Burgess N, O'Keefe J. Science. 2005; 308:873-876. [PubMed: 15879220]

68. Aronov D, Nevers R, Tank DW. Nature. 2017; 543:719-722. [PubMed: 28358077]

69. McKenzie S, Robinson NTM, Herrera L, Churchill JC, Eichenbaum H. J Neurosci. 2013; 33:10243-10256. [PubMed: 23785140]

70. Burke SN, et al. Hippocampus. 2011; 21:783-801. [PubMed: 21365714]

71. Fuhs MC, Touretzky DS. Neural Comput. 2007; 19:3173-3215. [PubMed: 17970649]

72. Hsieh LT, Gruber MJ, Jenkins LJ, Ranganath C. Neuron. 2014; 81:1165-1178. [PubMed: 24607234]

73. Manns JR, Eichenbaum H. Learn Mem. 2009; 16:616-624. [PubMed: 19794187]

74. McKenzie S, et al. Neuron. 2014; 83:202-215. [PubMed: 24910078]

75. Brown TI, Hasselmo ME, Stern CE. Hippocampus. 2014; 24:819-839. [PubMed: 24659134]

76. Tavares RM, et al. Neuron. 2015; 87:231-243. [PubMed: 26139376]

77. Dudek SM, Alexander GM, Farris S. Nat Rev Neurosci. 2016; 17:89-102. [PubMed: 26806628]

78. Mankin EA, Diehl GW, Sparks FT, Leutgeb S, Leutgeb JK. Neuron. 2015; 85:190-201. [PubMed: 25569350]

79. Alexander GM, et al. Nat Commun. 2016; 7:10300. [PubMed: 26806606]

80. Hitti FL, Siegelbaum SA. Nature. 2014; 508:88-92. [PubMed: 24572357]

81. Stevenson EL, Caldwell HK. Eur J Neurosci. 2014; 40:3294-3301. [PubMed: 25131412]

82. Eichenbaum H. Nat Rev Neurosci. 2014; 15:732-744. [PubMed: 25269553]

83. Guzowski JF, McNaughton BL, Barnes CA, Worley PF. Nat Neurosci. 1999; 2:1120-1124.

[PubMed: 10570490]

84. Kraus BJ, Robinson RJ II, White JA, Eichenbaum H, Hasselmo ME. Neuron. 2013; 78:1090-1101. [PubMed: 23707613]

85. Ranganath C, Hsieh LT. Ann NY Acad Sci. 2016; 1369:93-110. [PubMed: 27082833] 
86. Levy WB. Hippocampus. 1996; 6:579-590. [PubMed: 9034847]

87. Wallenstein GV, Eichenbaum H, Hasselmo ME. Trends Neurosci. 1998; 21:317-323. [PubMed: 9720595]

88. Wang Y, Romani S, Lustig B, Leonardo A, Pastalkova E. Nat Neurosci. 2015; 18:282-288. [PubMed: 25531571]

89. Czurkó A, Hirase H, Csicsvari J, Buzsáki G. Eur J Neurosci. 1999; 11:344-352. [PubMed: 9987037]

90. Salz DM, et al. J Neurosci. 2016; 36:7476-7484. [PubMed: 27413157]

91. Dragoi G, Buzsáki G. Neuron. 2006; 50:145-157. [PubMed: 16600862]

92. Maurer AP, Burke SN, Lipa P, Skaggs WE, Barnes CA. Hippocampus. 2012; 22:737-747. [PubMed: 21538659]

93. Geisler C, Robbe D, Zugaro M, Sirota A, Buzsáki G. Proc Natl Acad Sci USA. 2007; 104:8149_ 8154. [PubMed: 17470808]

94. Wikenheiser AM, Redish AD. Nat Neurosci. 2015; 18:289-294. [PubMed: 25559082]

95. Dennet DC, Kinsbourne M. Behav Brain Sci. 1992; 15:183-247.

96. Buzsáki, G., Llinás, R. Space and time in the brain Science. in the press;

97. Burak Y, Fiete IR. PLoS Comput Biol. 2009; 5:e1000291. [PubMed: 19229307]

98. Sanders H, Rennó-Costa C, Idiart M, Lisman J. Trends Neurosci. 2015; 38:763-775. [PubMed: 26616686]

99. Krupic J, Bauza M, Burton S, Barry C, O'Keefe J. Nature. 2015; 518:232-235. [PubMed: 25673417]

100. Chen G, Manson D, Cacucci F, Wills TJ. Curr Biol. 2016; 26:2335-2342. [PubMed: 27498565]

101. Hafting T, Fyhn M, Molden S, Moser MB, Moser EI. Nature. 2005; 436:801-806. [PubMed: 15965463]

102. Constantinescu AO, O'Reilly JX, Behrens TEJ. Science. 2016; 352:1464-1468. [PubMed: 27313047]

103. Buzsáki G, Moser EI. Nat Neurosci. 2013; 16:130-138. [PubMed: 23354386]

104. Buzsáki G, Peyrache A, Kubie J. Cold Spring Harb Symp Quant Biol. 2014; 79:41-50. [PubMed: 25752314]

105. Doeller CF, Barry C, Burgess N. Nature. 2010; 463:657-661. [PubMed: 20090680]

106. Kraus BJ, et al. Neuron. 2015; 88:578-589. [PubMed: 26539893]

107. Robinson NTM, et al. Neuron. 2017; 94:677-688.e6. [PubMed: 28434800]

108. Schlesiger MI, et al. Nat Neurosci. 2015; 18:1123-1132. [PubMed: 26120964]

109. Diehl GW, Hon OJ, Leutgeb S, Leutgeb JK. Neuron. 2017; 94:83-92.e6. [PubMed: 28343867]

110. Keene CS, et al. J Neurosci. 2016; 36:3660-3675. [PubMed: 27030753]

111. Hardcastle K, Ganguli S, Giocomo L. Nat Neurosci. 2017; 20:1474-1482. [PubMed: 29073649]

112. Wittlinger M, Wehner R, Wolf H. J Exp Biol. 2007; 210:198-207. [PubMed: 17210957]

113. Mittelstaedt, H., Mittelstaedt, ML. Papi, F., Wallraff, HG., editors. Homing by path integration. Avian Navigation. 1982. https://dx.doi.org/10.1007/978-3-642-68616-0_29

114. Redish AD, Rosenzweig ES, Bohanick JD, McNaughton BL, Barnes CA. J Neurosci. 2000; 20:9298-9309. [PubMed: 11125009]

115. Gothard KM, Hoffman KL, Battaglia FP, McNaughton BL. J Neurosci. 2001; 21:7284-7292. [PubMed: 11549738]

116. Touretzky DS, Redish AD. Hippocampus. 1996; 6:247-270. [PubMed: 8841825]

117. Redish AD, Touretzky DS. Hippocampus. 1997; 7:15-35. [PubMed: 9138665]

118. Golani I, Benjamini Y, Eilam D. Behav Brain Res. 1993; 53:21-33. [PubMed: 8466665]

119. Garvert MM, Dolan RJ, Behrens TE. eLife. 2017; 6:e17086. [PubMed: 28448253]

120. Hassabis D, Kumaran D, Vann SD, Maguire EA. Proc Natl Acad Sci USA. 2007; 104:1726-1731. [PubMed: 17229836]

121. Peters J, Büchel C. Neuron. 2010; 66:138-148. [PubMed: 20399735]

122. Foster DJ, Wilson MA. Nature. 2006; 440:680-683. [PubMed: 16474382] 
123. Davidson TJ, Kloosterman F, Wilson MA. Neuron. 2009; 63:497-507. [PubMed: 19709631]

124. Gupta AS, van der Meer MAA, Touretzky DS, Redish AD. Neuron. 2010; 65:695-705. [PubMed: 20223204]

125. Muller RU, Stead M, Pach J. J Gen Physiol. 1996; 107:663-694. [PubMed: 8783070]

126. O'Keefe J, Recce ML. Hippocampus. 1993; 3:317-330. [PubMed: 8353611]

127. Lubenov EV, Siapas AG. Nature. 2009; 459:534-539. [PubMed: 19489117]

128. Patel J, Fujisawa S, Berényi A, Royer S, Buzsáki G. Neuron. 2012; 75:410-417. [PubMed: 22884325]

129. Lisman JE, Idiart MA. Science. 1995; 267:1512-1515. [PubMed: 7878473]

130. Buzsáki G. Neuron. 2010; 68:362-385. [PubMed: 21040841]

131. Fernández-Ruiz A, et al. Neuron. 2017; 93:1213-1226.e5. [PubMed: 28279355]

132. Hasselmo ME, Bodelón C, Wyble BP. Neural Comput. 2002; 14:793-817. [PubMed: 11936962]

133. Colgin LL, et al. Nature. 2009; 462:353-357. [PubMed: 19924214]

134. Buzsáki, G. Rhythms of the Brain. Oxford University Press; 2006.

135. Place R, Farovik A, Brockmann M, Eichenbaum H. Nat Neurosci. 2016; 19:992-994. [PubMed: 27322417]

136. DeCoteau WE, et al. Proc Natl Acad Sci USA. 2007; 104:5644-5649. [PubMed: 17372196]

137. Hsieh LT, Ranganath C. Neuroimage. 2014; 85:721-729. [PubMed: 23933041]

138. Benchenane K, et al. Neuron. 2010; 66:921-936. [PubMed: 20620877]

139. Pfeiffer BE, Foster DJ. Nature. 2013; 497:74-79. [PubMed: 23594744]

140. Lisman JE, Otmakhova NA. Hippocampus. 2001; 11:551-568. [PubMed: 11732708]

141. Lisman JE. Prog Brain Res. 2007; 163:615-625. [PubMed: 17765741]

142. Pfeiffer BE, Foster DJ. Science. 2015; 349:180-183. [PubMed: 26160946]

143. Guzman SJ, Schlögl A, Frotscher M, Jonas P. Science. 2016; 353:1117-1123. [PubMed: 27609885]

144. Lisman JE, Talamini LM, Raffone A. Neural Netw. 2005; 9:1191-1201.

145. Royer S, Sirota A, Patel J, Buzsáki G. J Neurosci. 2010; 30:1777-1787. [PubMed: 20130187]

146. Colgin LL. Nat Rev Neurosci. 2016; 17:239-249. [PubMed: 26961163]

147. Lisman J, Buzsáki G. Schizophr Bull. 2008; 34:974-980. [PubMed: 18559405] 


\section{Box 1}

\section{Definitions}

\section{Episodic memory}

episodic memory refers to specific personal events, including information about where and when these events occurred.

\section{Rate remapping}

in rate remapping, the location of place fields and their relationship do not change, but the firing rate of the cell within the field changes (Fig. 2).

\section{Global remapping}

in global remapping, the relationship between place fields changes, distinguishing it from rate remapping (Fig. 2).

\section{Cognitive map}

a cognitive map is an internal representation of the layout of an environment, capturing what entities exist in the environment, where these entities are located relative to each other, and how these entities interact over the course of an event.

\section{Place cells}

cells found in the hippocampus that fire at a particular position in an environment, for example, the left corner of a room (Fig. 3).

\section{Grid cell}

cells found in the medial entorhinal cortex that fire at multiple (hexagonally spaced) positions in an environment (Fig. 4).

\section{Time cell}

time cells are neurons that fire at a particular moment in a temporally structured experience, tracking scalar time (Fig. 3).

\section{Context}

context refers to the background within which an event transpires. It can include spatial settings, time frames, and social situations, amongst others.

\section{Allocentric}

allocentric relations are those that exist between entities in an environment, independent of the organism and its movements.

\section{Consolidation}

Consolidation refers to a set of processes that stabilize learned information after its initial acquisition. It is both a synaptic-cellular process that undergoes a time-protracted transformation and a system-level process that can result in a restructuring of the 
information (for example, from episodic to semantic), reflecting a shift in the underlying neural substrates responsible for access to that information.

\section{Path integration}

Also known as dead reckoning, path integration maintains position within a coordinate system by updating that representation from cues signaling changes in position. Compare this to navigation from stable external cues, which can provide information about position directly (once learned; Fig. 4). 


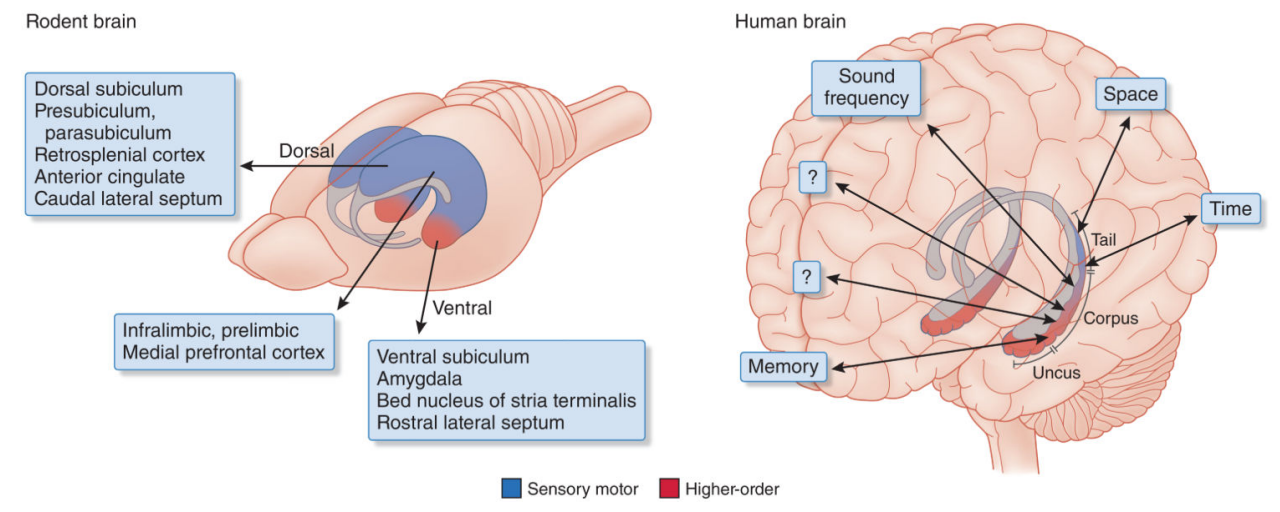

Figure 1.

Homologous regions of the hippocampus in the human and rat brains. The ventral quadrant of the rodent hippocampus became disproportionally enlarged in primates to keep up with the increasingly larger share of higher-order neocortex and formed the uncus and body. Only the relatively small tail part of the primate hippocampus communicates with visuospatial areas. This tail is the part that is homologous with the rodent dorsal-intermediate hippocampus. Differential connections to and from the different segments of the septotemporal axis are shown. Most recordings and manipulations in the rodent brain have been performed in the dorsal hippocampus. Adapted with permission from ref. 145, "Distinct representations and theta dynamics in dorsal and ventral hippocampus" 

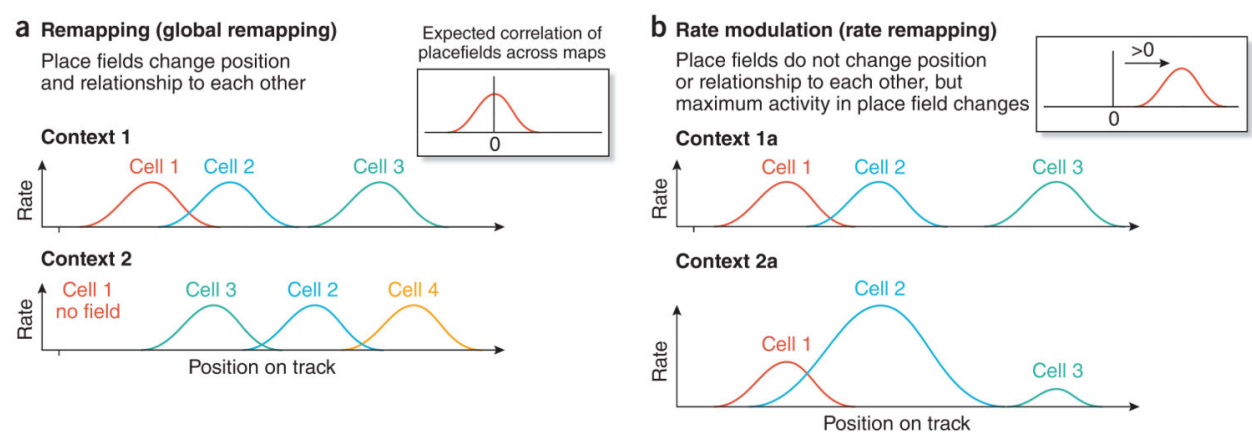

Figure 2.

Comparison of global remapping of hippocampal place cells to rate remapping. Place cell firing rate is plotted as a function of rat position along linear track. (a) Global remapping occurs in response to a context shift resulting from use of different tracks or the same track but with different behavioral relevance. Inset at right shows that the average position of place cell peak firing in one map is not correlated with peak position in the new map. (b) Rate remapping occurs following minor changes in context and produces changes in place cell firing rates but no change in the position of maximal activity. Inset at right shows that the average position of peak place cell firing in original map is highly correlated with peak position in the new map. 
a
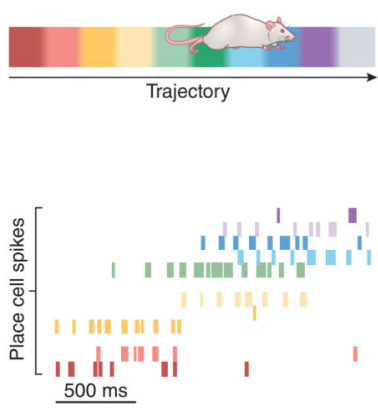

b
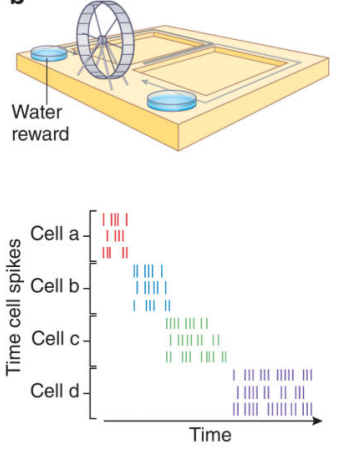

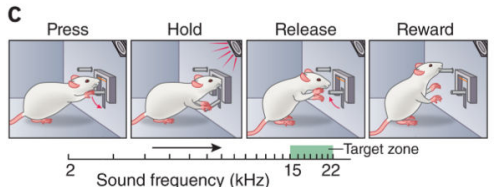

Sound frequency $(\mathrm{kHz})$
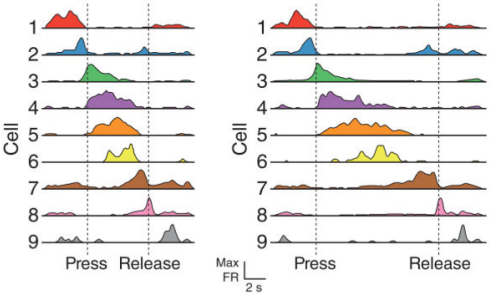

Figure 3.

Hippocampal pyramidal neurons provide organized responses to spatial stimuli, nonspatial stimuli, and time. (a) Top: in a simple navigation task, a rat runs back and forth along a linear track. Bottom: place cells, each represented by a different color, are activated as the rat runs along the track. Adapted with permission from ref. 146, Nature Publishing Group. (b) Top: rats alternated between left and right paths on a T-maze separated by running on a wheel. Bottom: time cells, each represented by a different color, are activated as time elapses during wheel running. Adapted with permission from ref. 82, Nature Publishing Group. (c) Top: in the sound-manipulation task depicted in a, rats learned to press and hold a joystick to increase the frequency of a pure tone until it reached a target frequency range, and to then release the joystick in order to obtain a reward. Bottom: CA1 place cells were active around particular frequencies, which resulted in sequences of firing fields that tiled the auditory frequency space. Their activity scaled with trial duration, contracting in fast trials (left) and expanding in slow trials (right). Similar results were obtained with grid cells. These results demonstrate that cells in the hippocampal formation can represent the gradually increasing frequency of a tone in a map-like fashion, like they do for other continuous dimensions such as space and time. Adapted with permission from ref. 68, Nature Publishing Group. 

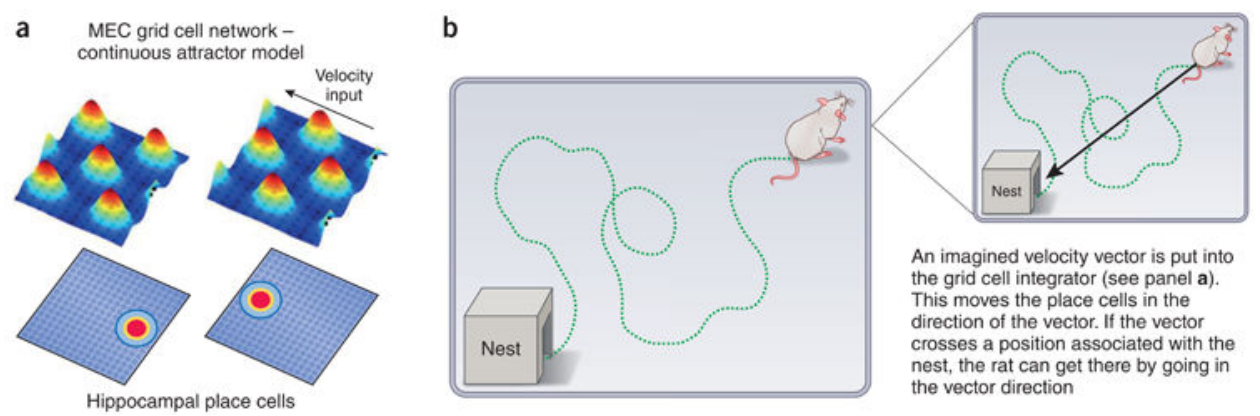

Figure 4.

Properties and utilization of grid cells. (a) Top left: grid-cell network in the MEC showing hexagonally spaced bumps of activity (red, peak rate). Either a true velocity signal or an imagined velocity signal is put into the grid-cell network. Top right: the bumps move leftward in the network in proportion to a true velocity vector derived from optic flow information, somatosensory information or vestibular information, thus performing an integration process. Cells in the network shown and those in similar networks but with different grid-cell spacing converge onto hippocampal cells and generate place cells (having maximal activity at a given position; bottom left. The position of active place cells moves left as result of the movement of activity bumps in the grid cell networks (bottom right). Adapted with permission from ref. 98, Elsevier. (b) Path integration occurs during a complex path taken by the animal, resulting in hippocampal place cell activity at a position that is directly relatable to the actual position of the animal even though no sensory information about the place was used in the process. Inset: one mechanism for finding a straight path to the rat's home is to generate an imagined velocity vector and put it into the grid cell network. This moves active place cells along the line of the arrow shown. If this activity leads to the excitation of 'home' place cells, the direction of the vector is the straightest direction of motion to get the rat home. 

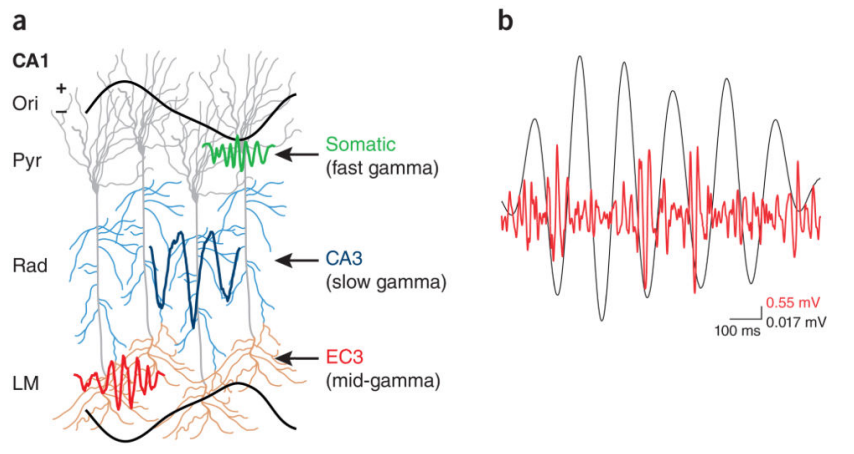

c

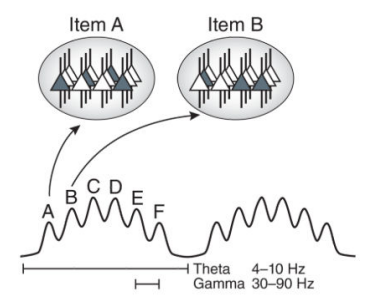

Figure 5.

Properties of theta and gamma oscillations in the hippocampus. (a) Dendritic layer gamma oscillations report activity of upstream neurons. Entorhinal layer 3 (EC3) mid-frequency gamma input (60-100 Hz) modulates distal dendrites in stratum lacunosum moleculare (LM) at the positive peak of CA1 pyramidal layer theta (Pyr), followed by CA3-projected slow-gamma $(30-60 \mathrm{~Hz})$ input in stratum radiatum $(\mathrm{Rad})$ on the descending theta phase. The gamma power ratios and their relative timing determine the theta phase of spiking of CA1 pyramidal cells. High-frequency $(90-150 \mathrm{~Hz})$ gamma power at the trough of the theta cycle (not shown) largely reflects spiking activity. Ori, stratum oriens. Adapted with permission from ref. 131, Elsevier. (b) Simultaneous filtered $(20-100 \mathrm{~Hz})$ intracellular recording from a CA1 pyramidal neuron (red) and extracellular recording (black) from the CA1 pyramidal layer. (c) Neural code organized by theta and gamma oscillations. Ovals at the top represent the shifting states of the network during two gamma cycles (spiking cells are dark gray and constitute the ensemble that codes for a particular item). Each gamma cycle contains a unique constellation of neurons (ensembles A to F). Adapted with permission from ref. 147, Oxford Academic Journals. 